Im großen und im kleinen immer wieder dasselbe Bild: Wo es darum geht, unter unsicheren Rahmenbedingungen umweltpolitische Entscheidungen zu treffen, melden sich die Politiker von der Bühne ab und überlassen das Geschehen den (Natur-)Wissenschaftlern. Scheinbar verschwindet das Politische aus dem Geschehen, das schließlich darüber bestimmt, welche Umweltqualität wir in unseren Dörfern, Regionen, Kontinenten und auf unserem Planeten dereinst haben werden. Ob der Weg nach Rio de Janeiro den längst fälligen Aufbruch der Politik aus ihrer selbstverschuldeten Unmündigkeit gegenüber der Wissenschaft bringt, ist angesichts der Haltung der Nord-Amerikaner zu bezweifeln. Dort scheint man immer noch abwarten zu wollen, bis die Wissenschaft den (unmöglichen) hundertprozentigen Beweis für bevorstehende Klimaänderungen erbracht haben wird.

\title{
Zur unzumutbaren politischen Rolle der Naturwissenschaften in der umweltpolitischen Debatte"
}

\author{
Peter Knoepfel*
}

\begin{abstract}
By means of four examples (curve diameter formula for the construction of highways; heavy metal standards for sewage sludges; evaluation of environmental impacts in environmental impact assessment procedures; evaluation of the greenhouse effect) important political implications within pretended scientific and technical research results are demonstrated. While politicians today often decide on pretty unimportant matters (for example, the introduction of the summer time and so on) crucial aspects of environmental quality are actually increasingly decided by the scientific community without any precise political mandate. Hence, real political substances within environmental policies seem to have disappeared.

But this is a misleading optical illusion. The present paper puts foreward some strategies aiming at making again appear
\end{abstract}

\footnotetext{
* Postadresse : Prof. Dr. P. Knoepfel Centre de recherche

Institut de hautes études en administration publique (IDHEAP)

route de Chavannes $31 \mathrm{~B}$

CH-1007 Lausanne (Schweiz)
}

1) Überarbeitete und erweiterte Fassung eines Vortrags am Tag "Umwelt" des ETH-Forums für Umweltfragen in Zürich am 15. Januar 1991.

2) Unter dem "Politischen" verstehen wir in diesem Aufsatz diejenige Substanz einer Entscheidung die eindeutig und legitimerweise je nach der vorgenommenen Interessenbewertung zugunsten der einen oder anderen sozialen oder ökologischen Gruppe einen unterschiedlichen Gehalt aufweisen wird und soll. these still existing political contents within "technizised" environmental policies. The political responsibility for these political contents must be assumed by those being democratically elected to bear the burden of political risks, namely the politicians. Whenever natural or social sciences should assume this heavy responsibility they inevitably run the risk of loosing their independency.

Keywords: environmental impact assessment, environmental policy, legitimacy, political responsibility, scientific community, "technizised" policies

\section{Einleitung: \\ Naturwissenschaftliche Kleider der Politik}

Parlamentarier, die sich für die Einführung von Grenzwerten für cancerogene Luftschadstoffe einsetzen, werden oft darauf verwiesen, daß natur- und medizinalwissenschaftliche Grundlagen für den Erlaß solcher Grenzwerte gegenwärtig fehlten. Es sei Sache der Wissenschaft und nicht der Politik, solche Grenzwerte zu erarbeiten. Ähnlich geht es etwa Unternehmern, die sich bei der Verwaltung danach erkundigen, welche Schadstoffe sie mit welchem Reinigungsgrad aus der Abluft entfernen müssen. Sie werden bei neuen Schadstoffen mit- unter mit der lakonischen Antwort beschieden, dies sei in ihrem Falle nicht von vornherein festlegbar; die Wissenschaft würde sich im gegebenen Moment dazu "dann schon" äußern. Ist dieser gegebene Moment vielleicht dann gekommen, wenn neue Großinvestitionen getätigt und die Maschinen einsatzbereit sind?

So scheinen es denn die Ärzte, Biologen, Chemiker oder Physiker zu sein, die in ihren "wissenschaftlichen" Labororatorien gesellschaftlich bindende Entscheidungen über die Qualität unserer natürlichen, baulichen und damit auch sozialen Umwelt fällen. Politik hat da so will es scheinen - nichts mehr zu melden. Je mehr sich unsere großen öffentlichen Politiken "vernaturwissenschaftlichen", desto weniger sollen $\mathrm{Re}$ gierungen, Parlamente oder das Volk als demokratisch legitimierte Instanzen unserer Politik dazu zu sagen haben. Es will somit scheinen, als ob zumindest in den seit den siebziger Jahren geschaffenen, stark "technizistischen" Politiken das Politische ${ }^{2)}$ allmählich abstirbt. Sollte sich dieser erste Eindruck bestätigen, so müßte sich auch die politisch wertende Beschäftigung mit Politik jedenfalls für den Bereich dieser neueren Politiken als überflüssig erweisen.

Diese Diagnose teile ich nicht. Ich behaupte vielmehr, daß das Politische nicht verschwindet oder abstirbt. Es hat sich in den letzten zehn bis fünfzehn Jahren nur in seiner äußeren Erscheinungsform verwandelt, ohne indessen die Qualität geändert zu haben: Um Macht und Einfluß auf gesellschaftlich verbindliche Entscheidungen wird auch in jenen technizistischen oder "vernaturwissenschaftlichten" Politiken gerungen; geändert haben sich allerdings die Sprache und die Orte der Auseinandersetzung. Das Politische trägt heute mehr denn je naturwissenschaftliche Kleider. Es artikuliert sich weniger in parteipolitischen Farben als vielmehr in Mikrogrammen, statistischen Zahlen und mathematischen Kurven. Zur Beweisführung werden im folgenden Abschnitt vier Beispiele angeführt werden, die anschließend vertiefend analysiert

\section{Vier Beispiele}

\section{Technische Normung ${ }^{[1]}$}

Die Formeln, nach denen Kurvenradien für Nationalstraßen berechnet werden, sind in keinem förmlichen $\mathrm{Ge}$ setz geregelt. Sie gehören zum allgemeinen Ingenieurwissen und werden den 
angehenden Straßenbauingenieuren an den Schulen vermittelt; sie stellen das Ergebnis von komplizierten physikalischen, materialtechnischen et cetera Berechnungen und Erfahrungswerten dar und werden im Straßenbau täglich angewendet ${ }^{[2 \mid}$.

Bekanntlich hat nun aber ein engerer oder weiterer Kurvenradius mitunter beachtliche Auswirkungen auf den Raumbedarf von Straßenbauten, auf die dem Bau zu opfernden landwirtschaftlichen Nutzflächen und oft auch auf Landschafts- und Siedlungsstrukturen ${ }^{[3]}$. Die Formeln sind daher von erheblicher raumplanerischer und somit auch gesellschaftspolitischer Bedeutung. Jeder Naturwissenschafter wird für selbstverständlich halten, daß eine solche Formel je nach der ins Auge gefaßten maximalen Fahrgeschwindigkeit variieren wird. Nach Auskunft von Fachleuten (SIA) ist der Standardformel eine Höchstgeschwindigkeit von ca. $180 \mathrm{~km} / \mathrm{h}$ zugrunde gelegt worden. Dieser Wert hat nie in einem Parlament zur Debatte gestanden. Paradoxerweise wurde indessen etwa das Schweizervolk unlängst zu den Urnen gerufen, um über die Festsetzung von Höchstgeschwindigkeiten auf den Straßen auf Verfassungsstufe abzustimmen ${ }^{[4]}$. Wenn man bedenkt, daß die tatsächliche Fahrweise eher von den baulichen Gegebenheiten und weniger von der Straßensignalisation abhängt, wird man sich zu Recht die Frage nach dem Sinn derartiger Volksabstimmungen stellen. Wird da nicht über etwas abgestimmt, das faktisch von den Ingenieuren längst entschieden wurde?

\section{Umweltverträglichkeitsprüfung}

In den spätestens seit dem 1. Januar 1989 für größere Werke regelmäßig erstellten Umweltverträglichkeitsberichten muß der Planungsträger bekanntlich aufzeigen, welche Auswirkungen sein Vorhaben auf die Umweltqualität haben wird ${ }^{[5]}$. Er hat diese Auswirkungen auch zu bewerten. Diese Bewertung muß er nach Maßgabe der gesetzlichen Bestimmungen und namentlich der einschlägigen Immissionsgrenzwerte vomehmen ${ }^{[6]}$. Nun ist allerdings bekannt, daß solche Grenzwerte nur für sehr wenige Umweltqualitätsparameter gesetzlich geregelt sind. Für eine Vielzahl (bekannter und unbekannter) Schadstoffe fehlen solche Grenzwerte und Bewertungskriterien. Falls sich der Umweltverträglichkeitsbericht mit diesen Stoffen überhaupt auseinandersetzt, ist das bearbeitende Planungsbüro auf naturwissenschaftliche Erkenntnisse, Daten und Angaben über Wirkungszusammenhänge in der Literatur angewiesen. Früher war das übrigens auch für jene Schadstoffe der Fall, für die heute gesetzlich festgesetzte Grenzwerte verfügbar sind.

Dabei stellen sich viele Ungewißheiten ein. Diese ergeben sich in Politik und Öffentlichkeit bekanntlich vor allem dann, wenn die herangezogenen Grenzwerte etwa im internationalen Vergleich sehr streng sind. Ein solcher Grenzwertvergleich zeigt im übrigen, daß die Wissenschaftler Umweltqualität beziehungsweise die Anforderungen an die menschliche Gesundheit und an die Überlebensfähigkeit von Ökosystemen von Land zu Land recht unterschiedlich ansetzen ${ }^{[7]}$.

Solche Bewertungen der Umwelteinwirkungen in UVP-Berichten ${ }^{[4]}$ haben erhebliche Auswirkungen auf das Ausmaß der notwendigen Schutzmaßnahmen. Darüber hinaus kommt ihnen ein beachtliches politisches Gewicht bei der formellen Genehmigung oder Ablehnung einer der geplanten Anlage zu. Daß UVP-pflichtige Großanlagen ihrerseits auch gesellschaftliche Umwelten und sozio-kulturelle Landschaften nachhaltig prägen, muß im Zeitalter scharfer Konkurrenz um den knapp gewordenen Raum und einer zunehmenden ökologischen Sensibilisierung der Öffentlichkeit nicht besonders begründet werden ${ }^{[8]}$.

\section{Treibhausgase}

Zur Vorbereitung der zweiten Weltklimakonferenz hatte die Deutsche Bundesregierung am 13. Juli 1990 das weltweit bisher wohl umfangreichste und (neben Japan) konkreteste Handlungsprogramm für eine 25 prozentige $\mathrm{Re}$ duktion der $\mathrm{CO}_{2}$-Emissionen in Deutschland bis zum Jahr 2005 (bezogen auf die Emissionen von 1987) vorgelegt. Diesem immer noch gültigen Dokument liegen folgende Überlegungen zugrunde: »- In Kenntnis der wissenschaftlichen Ungewißheit über Ausmaß und Zeitpunkt global und regional negativer Effekte der Klimaveränderung sollte die Bundesregierung nach dem Vorsorgegrundsatz handeln, das heißt die verfügbaren $\mathrm{CO}_{2}$ Minderungspotentiale soweit wie möglich ausschöpfen.

- Vorteil einer solchen Vorsorgestrategie ist die Vermeidung krisenbedingter Anpassungskosten, denen sich die Bundesrepublik Deutschland - auch wenn sie regional von den einschneidenden Problemen von Klimakatastrophen nicht unmittelbar betroffen sein sollte - aufgrund ihrer internationalen wirtschaftlichen Verflechtung und ihrer politischen Solidarverantwortung für die weltweite Entwicklung nicht entziehen kann.« ${ }^{[9]}$ Der umfangreiche Maßnahmenkatalog, der auch eine $\mathrm{CO}_{2}$-Abgabe beinhaltet, sieht drastische Eingriffe vor in den Bereichen Haushalte/Kleinverbrauch (Gesamtbeitrag: $10.5 \%$ ), Energieversorgung (Gesamtbeitrag um $6 \%$ ) und Industrie (Gesamtbeitrag: $5.5 \%$ ). Mit einer Förderung erneuerbarer Energien, Maßnahmen im Verkehr und in der Abfallwirtschaft sollen weitere Reduktionen (insgesamt um $10 \%$ ) erzielt werden. Diese einschneidenden Maßnahmen, die vermutlich zu einer dramatischen Veränderung der deutschen Energiegesellschaft führen werden, wurden trotz des Umstandes beschlossen, daß gegenwärtig noch »... nicht alle komplizierten naturwissenschaftlichen Zusammenhänge einer Umweltbedrohung im einzelnen geklärt sind «. Denn die Vorsorge gebiete, " ... in unserem eigenen Interesse und im Interesse künftiger Generationen, bereits jetzt zu handeln, unter anderem wegen der langen zeitlichen Verzögerung, die zwischen der Entstehung von Treibhausgasen und ihren Auswirkungen auf das Klima liegen ${ }^{101}$. Ein ähnliches (allerdings etwas weniger weitgehendes) Programm soll auch die Schweiz verabschieden ${ }^{[11]}$.

Demgegenüber hat der Präsident der Vereinigten Staaten von Amerika die US-Delegation mit der Botschaft an die besagte Klimakonferenz gesandt, der wissenschaftliche Nachweis von KlimaAuswirkungen der Treibhausgase und insbesondere der $\mathrm{CO}_{2}$-Emissionen sei gegenwärtig nicht erbracht, weshalb das Ergreifen entsprechender Maßnahmen heute verfrüht wäre. Diese Reaktion desjenigen Landes, das für mehr als ein Fünftel der $\mathrm{CO}_{2}$-Emissionen verantwortlich ist $(22.3 \%)$, und dessen Einwohner mit 20 Tonnen $\mathrm{CO}_{2}$-Emissionen pro Jahr eine weltweit einsame Spitzenposition einnehmen (Bundesrepublik Deutschland: 11.7 Tonnen) ${ }^{[12]}$, entlarvt sich als Ausweichmanöver und Kniefall der Politik vor einer mächtigen und sehr eigensüchtigen Wirtschaft. Wie die Deutsche Bundesregierung zu Recht betont, kann die Verantwortung für das Handeln gegen drohende globale Klimaschäden nicht den Naturwissenschaften und ihrer Vorstellung von einer exakten Beweisführung überlassen werden.

\section{Klärschlamm}

In den letzten Jahren haben die Gemeinden landauf, landab ganz nach dem Gebot des Bundes eifrig Kläranlagen gebaut. Heute werden in der Schweiz fast $90 \%$ der Siedlungs- und Industrieabwässer in solchen Anlagen gereinigt [13]. Eine Kläranlage ist aber kein Wundertäter, der den herbeigeführten 
Schmutz einfach in ein Nichts transformieren würde. Je besser die Anlage funktioniert, desto mehr Schadstoffe hält sie zurück. Diese finden sich ihrerseits unter anderem in entsprechend kontaminierten Klärschlämmen wieder. Wohin mit diesem Schlämmen?

Die Wissenschaft stiftet auch hier nur relative Gewißheit. Sofern der Schwermetallgehalt bestimmte Grenzwerte nicht überschreitet, können die Schlämme angeblich problemlos landwirtschaftlich verwertet werden. Die Grundlage der Berechnung der Schwermetallgrenzwerte, die die landwirtschaftlichen Forschungsanstalten in der zweiten Hälfte der siebziger Jahre für die Schweiz festlegten, ist die - ebenfalls in keinem gesetzgeberischen Dokument ausdrücklich erwähnte - Annahme, daß die Böden bei einer regelmäßigen Klärschlammaustragung während einer Dauer von rund 50 Jahren keine schwermetallbedingten Schäden erleiden sollten ${ }^{[14]}$. 50 Jahre - und danach? In gewissen Gebieten ist die Halbzeit bereits überschritten. Über die Qualität der Böden unserer Kinder befinden offenbar Forschungsanstalten, während sich unsere Parlamente mit angeblich weit wichtigeren Fragen, wie der Wahl von Parlamentspräsidenten oder mit der Einführung der Sommerzeit, herumschlagen.

\section{Vertiefende Betrachtung: Der Dreischritt "Politik - Wissenschaft - Politik"}

Aus der Sicht desjenigen, der sich mit dem Politischen befaßt, geschieht in den vier erwähnten Beispielen ein generalisierungsfähiger Dreischritt:

\section{Von der Politik zur Naturwissenschaft}

Die Verwaltung oder - in selteneren Fällen - das Parlament stellt fest, daß in einem bestimmten Bereich ein ökologisches Problem besteht. Dieses soll im Hinblick auf die Sicherung der Funktionsfähigkeit des davon betroffenen gesellschaftlichen Bereichs unbedingt irgendwie gelöst werden, denn es stiftet unter den beteiligten Akteuren Unsicherheit. Bald zeigt sich, daB das Problem eine Folge fast unüberschaubar vieler iterativ vernetzter Einflußfaktoren ist. Es liegt eine Komplexität vor, die nur durch naturwissenschaftliche Klärung erhellt werden kann. Regierung und Verwaltung übergeben das Problem deshalb der "scientific community".

Diese Wissenschaftsgemeinschaft soll nun dafür sorgen, daß Straßen umwelt- schonend gebaut, die Umwelteinwirkungen von wichtigen Großanlagen unter Kontrolle gehalten oder die Umweltverträglichkeit des Klärschlammaustrags gesichert werden können. Dabei bleibt die Fragestellung meist recht vage; Regierungen und Verwaltungen sind oft kaum imstande, besonders interessierende Problemursachen auszumachen. Unter solchen Bedingungen wird sich die Wissenschaft ihrerseits auf jene Problemursachen konzentrieren, für die exakt meßbare Parameter bekannt sind. Unbekanntes läßt sich mit bekannten Methoden nur schwer entdecken. Auf Fragen, die man nicht stellt, erhält man keine Antwort. Ursachen, für die es keine Meßinstrumente gibt, bleiben im Dunkeln. Gesellschaftliche Ursachen für die eingetretenen Veränderungen werden dabei ebenfalls oft ausgeblendet ${ }^{[15]}$.

Man mag nun sagen, dies sei ein ganz normaler Prozeß ohne politische Implikationen, weil es doch nur darum gehe, "Entscheidungsgrundlagen" zu erarbeiten. Politik und Verwaltung seien immer noch frei, die von der Wissenschaftsgemeinschaft vorgetragenen $\mathrm{Er}$ gebnisse zu akzeptieren oder nicht. Das trifft indessen nicht zu: Denn das Politische an diesem Vorgang liegt darin, $\mathrm{da} ß$ sich die politischen Instanzen mit dieser Generalübertragung des Themas an die Wissenschaft der Möglichkeit begeben, auf den politischen Gehalt der Fragestellung Einfluß zu nehmen. Dadurch rennen Politik und Verwaltung gewissermaßen ohne Zwischenhalt vom Orakel zu Delphi oder vom Papst zu Rom direkt in die Labors auf dem Hönggerberg, ohne das - aufklärerische Recht zu beanspruchen, auf die Problemdefinition Einfluß zu nehmen. Probleme definieren aber heißt gleichzeitig Problemlösungen vorstrukturieren und damit nachhaltig beeinflussen ${ }^{116]}$. Dieser quasi freiwillige Verzicht der Politik auf Mitbestimmung des Politischen ist um so bedenklicher, als bei den nunmehr inthronisierten Naturwissen-schaften eine "ungeheuerliche Leichtigkeit im Umgang mit dem Politischen" (Baccini ${ }^{[17]}$ ) besteht.

So wäre in unserem ersten Beispiel (Abschnitt 2) etwa zu fragen, für welche Verkehrsteilnehmer mit welchen Verkehrswünschen und -gewohnheiten StraBen gebaut werden sollen. Wer soll diese Gewohnheiten definieren? Die Automobilhersteller oder die Fahrzeughalter mit ihren Sicherheitsbedürfnissen? Oder sollten dazu auch die betroffenen stummen Wesen der Natur etwas zu sagen haben?

\section{Vom Problem zur}

\section{(natur-)wisssenschaftlichen Lösung}

Nach dieser Generalübertragung politischer Entscheidungskompetenzen erarbeitet die Wissenschaftergemeinschaf Lösungen: Mit diesen sollen ökologische Ungewißheiten abgebaut werden, damit soziale, für politisch-administratives Handeln relevante Gewißheiten entstehen ${ }^{[181}$. Diese Lösungen geben Bedingungen an, bei deren Einhaltung mit einer gewissen Sicherheit vorherbestimmbare Ergebnisse eintreten. Wenn der Kurvenradius nach Maßgabe der Formel berechnet wird, dann ist zu erwarten, daß das Großsystem "Straße" funktionsfähig ist. Unfälle, Materialabnutzungen und so weiter lassen sich dadurch voraussichtlich vermeiden, und die Mobilität ist gewährleistet. Wenn zur Behebung der in der UVP ${ }^{[5]}$ angegebenen Umweltbelastungen die vorgeschlagenen Maßnahmen getroffen werden, dann ist nach dem gegenwärtigen Stand des Wissens davon auszugehen, daß die geplante Neuanlage die Funktionsfähigkeit des betroffenen Ökosystems nicht beeinträchtigt. Wenn die Landwirte nur Klärschlamm ausbringen, dessen Schwermetallkonzentrationen sich unterhalb des Grenzwertes bewegen, bleibt nach dem gegenwärtigen Stand des Wissens das landwirtschaftliche Produktionssystem "intakt".

Auch bei solchen "Lösungen" ist das Politische präsent. Es stellt sich sowoh auf der "Wenn-" als auch auf der "DannSeite" ein: Auf der (konditionalen) Wenn-Seite manifestiert es sich in Wertungen und der Kontrollierbarkeit der Bestimmungsfaktoren für bestimmte Problemkonstellationen. Dabei stellt sich immer wieder die - bekannte - Frage nach den durch Naturereignisse oder menschliche Einwirkungen möglicherweise verbleibenden Restrisiken ${ }^{[19]}$.

Auf der "Dann-Seite" stellt sich die politisch wohl noch bedeutsamere Frage, was denn als "Funktionsfähigkeit" des von Politik und Verwaltung als "gestört" bezeichneten Systems anzusehen ist. Wer definiert, was funktionsfähig, was "störungsfrei" ist? Bezieht sich Funktionsfähigkeit auf das angeblich gestörte Teilsystem, zum Beispiel auf die Verkehrsfähigkeit einer Straße oder auf die Abwesenheit von physisch meßbaren Belastungen in der näheren oder weiteren Umgebung einer UVP-pflichtigen Anlage? Ist "funktionsfähig" auch jener Planet, dessen aquatische Systeme und potentiellen Klimakatastrophen durch technische Vorkehrungen wie Dämme, Lawinenverbauungen et cetera künstlich im Gleichgewicht gehalten werden? Oder 
schließt Funktionsfähigkeit auch Abwesenheit von Störungen in mittelbaren und unmittelbaren Nachbarsystemen des Problemsystems ein? $\mathrm{Zu}$ denken wäre etwa an Lärm-, Luft- und Bodenbelastungen sowie Raumverzehr und Bodenversiegelung in den benachbarten Ökosystemen entlang einer Straße, an großräumige Auswirkungen einer UVPpflichtigen Anlage infolge von Schadstoffverfrachtungen, an langfristige Bodenbelastungen klärschlammgedüngter Felder oder an das zwangsläufig vertiefte Nord-Süd-Gefälle, würden von Entwicklungs- und Industrieländern gleichermaßen lineare Reduktionen der $\mathrm{CO}$,-Emissionen verlangt.

Hier stellt sich ferner auch die Frage nach der sozialen beziehungsweise ökologischen Finalität von "Funktionsfähigkeit": Sollen im Mittelpunkt der Mensch (anthropozentrische Betrachtungsweise) oder die Ökosysteme insgesamt stehen (ökozentrische Betrachtungsweise) ${ }^{20]}$ ? Oder: Funktionsfähig für welche sozialen Gruppen [2]]? Soll etwa die Funktionsfähigkeit eines Straßensystems an den Bedürfnissen der vergleichsweise kleinen und wirtschaftlich starken Verkehrsteilnehmergruppe gemessen werden, die sich Großlimousinen leisten kann, die problemlos mit $180 \mathrm{~km} /$ $\mathrm{h}$ durch die Landschaft rasen, oder etwa am Flächenbedarf der betroffenen Landwirte? Sollen die Schutzmaßnahmen bei UVP-pflichtigen Anlagen primär gute Wohnlagen verhältnismäßig wohlhabender Anrainer oder die Erholungsgebiete der Gesamtbevölkerung schützen? Oder soll Boden vor Schwermetallbelastungen im Interesse der wirtschaftlichen Belange der Landwirte, im Interesse der (heutigen/zukünftigen) Nahrungsmittelkonsumenten oder im Interesse der ökologischen Funktionen der Bodenmikroorganismen geschützt werden? Sollen Klimaschutzmaßnahmen im Interesse der bedrohten Humangesellschaften oder auch im Hinblick auf den Ökosystemschutz getroffen und damit erheblich frühzeitiger und weitergehend konzipiert werden, als dies gegenwärtig auf höchster Ebene diskutiert wird?

All dies sind hochpolitische Optionen, die einer politischen Antwort bedürfen; die Verantwortung dafür kann nicht der bereits zitierten "ungeheuerlichen (politischen) Leichtigkeit der Naturwissenschafter" (Baccini) überlassen bleiben. Hinzu kommt schließlich eine kollektive Bewertung der Anforderungen an die Exaktheit der Beweisfiihrung für angebliche Wirkungszusammenhänge einer Ursache-Wirkungskette. Namentlich bei nicht-linearen Systemfunktionen können nach gesicherten Erkenntnissen der Naturwissenschaften mitunter ein Kollaps oder ein Chaos eintreten, das kaum vorhersehbar ist ${ }^{[221}$. Welchen Sicherheitsgrad wollen wir? Wie lange will die Gesellschaft warten, bis die Naturwissenschaft uns angeblich nachgewiesene, hundertprozentig richtige UrsacheWirkungs- Zusammenhänge nachweist? Was soll bis zu jenem Zeitpunkt gelten? Lassen sich Nicht-Wirkungen wissenschaftstheoretisch und praktisch überhaupt nachweisen ${ }^{[23 \mid}$ ?

All diese - bekannten - Fragen kulminieren schließlich in der nur politisch beantwortbaren Frage nach notwendigen Sicherheitsmargen bei Unsicherheiten, für die ebenfalls keinem Naturwissenschafter die kollektive Verantwortung übertragen werden kann. Das wird vor allem auch bei der $\mathrm{CO}_{2}$-Problematik deutlich: Die Administration Bush scheint bei ihrer Suche nach solchen Naturwissenschaftlern erfolgreich gewesen zu sein, die ihr den "Beweis des Nicht-Beweises" eines Zusammenhangs von globaler Erwärmung / Klimaänderungen und Treibhausgas-Emissionen geliefert haben. Die wissenschaftlichen Ungereimtheiten seien so groß, daß auch das Nichtergreifen von $\mathrm{CO}_{2}$-bezogenen Maßnahmen genügend Sicherheit vor globalen Klimaänderungen böten. So ließen sich denn einmal mehr die Amerikaner und Amerikanerinnen ihren weltweit einmalig hohen Konsum von Benzin und Energie sicherstellen. Mit diesem wissenschaftlichen Trick ist das Zirkuskarussell auch für die nächste BillionenDollar-Wahl einmal mehr bestens geölt.

\section{Die politisch-administrative Rezeption}

Politik und Verwaltung verlangen von den Naturwissenschaftern zunehmend, $\mathrm{daB}$ sie realisierbare Lösungsvorschläge unterbreiten. Damit wird ihnen auch eine dritte kollektive und somit politische Verantwortung übertragen, die sie allein nicht tragen können. Die Folge davon ist, daß sie in ihrem Denken zunehmend politiknaher werden ${ }^{[24]}$ und somit bei ihren Vorschlägen das "politisch Mögliche" anstreben. Daraus resultiert nicht selten eine oft (nicht explizite) selbstzensurierende Vorwegnahme des politischen Entscheides und damit eine Einengung des Horizonts möglicher Lösungen. Auf der Suche nach der Kurvenradiusformel wird stillschweigend davon ausgegangen, da $B$ etwa eine Fixierung der Höchstgeschwindigkeit auf $100 \mathrm{~km} / \mathrm{h}$ politisch nicht tragbar wäre. Die Vorschläge für Maßnahmen zur Reduktion von Umweltbelastungen UVP-pflichtiger Anlagen tragen in vielen Fällen bereits den Stempel eines politisch ausgehandelten Kompromisses; angeblich allzuweit gehende Schutzmaßnahmen werden in den Szenarios schon gar nicht mehr diskutiert. Die Grenzwerte für Schwermetalle in den Klärschlämmen setzen stillschweigend voraus, daß etwa Maßnahmen in den Haushalten oder an den schwermetallhaltigen Produkten selbst politisch kaum durchsetzbar wären, weshalb sich diese Stoffe auch weiterhin in den Schlämmen finden werden; deshalb müsse ein Kompromiß zwischen den Belangen der Betreiber von Kläranlagen und der Landwirte gefunden werden ${ }^{[25]}$. Man spricht heute denn auch mehr denn je von den berühmten "politischen Rahmenbedingungen", die der Naturwissenschaftler stillschweigend zu akzeptieren hat.

\section{Zur neuen Rolle von Sozialwissenschaften, Naturwissenschaften und Politik bei der Wiedereinsetzung des Primats von Politik im Bereich des Politischen innerhalb der "vernaturwissenschaftlichten" Umweltdebatte}

Die hier nur in groben Zügen vorgetragene Analyse zeigt, daß das Politische nicht verschwindet, sondern im Laufe dieses Dreischrittes lediglich in andere Kleider schlüpft. Wer ein hinreichend geschultes Auge für dieses Politische hat, wird es auch nach erfolgter Metamorphose wieder entdecken. Daraus wird unmittelbar einsichtig, daß den Naturwissenschaften in diesem Arrangement eine allzu hohe politische Verantwortung übertragen wird, die sie auch beim besten Willen nicht zu tragen imstande sind. Diesbezüglich im Gegensatz zu den Vertretern einer gesellschaftlich und politisch verantwortlichen Wissenschaft ${ }^{[26]}$, bin ich der Meinung, daß diese auch einer noch so gesellschaftsbewußten Wissenschaftergemeinschaft nicht aufgebürdet werden kann und darf. Im demokratischen Staat gehört die Verantwortung für das Politische denjenigen übertragen, die das Volk dafür gewählt hat.

\section{Zur neuen Rolle der Sozialwissenschaften}

Bei dieser Wiedereinsetzung des Primats der Politik kommt auch den Sozialwissenschaften ein wichtiger Part zu. Dieser besteht in:

- einem kritischen Sichtbarmachen des verdeckten Politischen in den Frage- 
stellungen oder "Nicht-Fragestellungen", in den Bewertungen von Unsicherheiten und Sicherheitsmargen ("Wenn-Seite") und in den Definitionen dessen, was unter dem Begriff der wiederherzustellenden Funktionsfähigkeit der tangierten Systeme angesehen wird ("Dann-Seite"). Dieses Aufdecken oder Wiederentdecken des Politischen im naturwissenschaftlich-technischen Diskurs halte ich für die wichtigste theoretische und praktische Aufgabe einer modernen Politikwissenschaft, die sich nicht nur mit politischen Ritualen, sondern mit tatsächlich politisch relevanten Entscheidungsabläufen befassen will. Diese Funktion wird sie nicht zuletzt auch im Interesse der von den jeweiligen Entscheidungen betroffenen Gruppen wahrzunehmen haben, die infolge fehlender Sachkenntnisse und finanzieller Mittel nicht in der Lage sind, sich und ihre Interessen im naturwissenschaftlich-technizistischen Gestrüpp wiederzuerkennen. Denn Macht und Ohnmacht, Einfluß und Marginalisierung finden zumindest bei solchen Politiken ihren Niederschlag in Kurvenradienformeln, UVP-Berichten, Milligrammangaben oder in der Bewertung von Risiken, Unsicherheiten und Sicherheitsmargen in der Klimaforschung ${ }^{[26 \mathbf{b}] \text {; }}$

- der Mitwirkung an der Problemdefinition bezüglich der sozialen und politischen Bestimmungsfaktoren einer Problemkonstellation. Dies könnte der wesentlichste Beitrag der Sozialwissenschaften zu einer interdisziplinären Projektarbeit sein. Eine Bewährungsprobe werden die Sozialwissenschaften in der Schweiz im Rahmen des Nationalen Forschungsprogramms "Klimaänderungen und Naturkatastrophen" (NFP 31) $\mathrm{zu}$ bestehen haben, wo auch gesellschaftliche Auswirkungen klimabedingter Ökosystemveränderungen sowie zu erwartender Naturkatastrophen und dagegen vorzusehende politische und gesellschaftliche Strategien untersucht werden ${ }^{[27]}$;

- der adäquaten Verortung des sichtbar gemachten Politischen im institutionellen politischen Raum. Das "Zurückholen" des Politischen in politische Entscheidungsprozesse und die Erarbeitung angemessener Entscheidungsstrukturen im Rahmen eines demokratischen Staatswesens gehören zu den ureigenen Funktionen der schweizerischen Politikwissenschaft. Dieses Feld kann nicht den Verfassungsjuristen überlassen werden, die hier allzuoft von schematischen und formalistischen Konzepten überholter Normenhierarchien und hochgradig hierarchisch strukturierten Institutionen ausgehen. Gefordert ist nicht weniger als eine fruchtbare Verkoppelung von institutioneller Phantasie und technischen Sachkenntnissen ${ }^{[28]}$;

- der Beteiligung am naturwissenschaftlichen Problemlösungsprozeß. Dabei müßten sie insbesondere bei den kritischen Übergangsphasen der Formulierung der Fragestellung vom politischen an den naturwissenschaftlichen Raum, bei der Erarbeitung der Problemlösungen nach dem "Wenn-DannSchema" und bei der Umformulierung von naturwissenschaftlichen Problemlösungsszeniarios in politisch-administrativ handhabbare Regelungsvorschläge mitdenken. Es geht dabei wohlgemerkt nicht um ein Ersetzen der politischen Wertungen und Bewertungen der Naturwissenschafter durch jene der Politikwissenschafter. Denn eine solche käme ebenso wie die erstere einer politischen Bewertung durch noch unberufenere Zensoren gleich. Im Mittelpunkt steht vielmehr eine Reflexion und schließlich ein Sichtbarmachen des Politischen im naturwissenschaftlichen Forschungsprozeß zuhanden der politischen Entscheidungsträger.

\section{Zur neuen Rolle der Naturwissenschaften}

Als Sozialwissenschafter bin ich nicht berufen, den Naturwissenschaftern ihre Rolle bei der Wiedereinsetzung des Primats der Politik im "vernaturwissenschaftlichten" Umweltdiskurs zu definieren. Deshalb beschränke ich mich hier auf eine grundsätzliche Betrachtung: Es wäre verfehlt, aus der vorausgegangenen Analyse den Schluß zu ziehen, die Naturwissenschaften müßten sich wiederum vermehrt einer angeblichen wissenschaftlichen Wertfreiheit verschreiben und sich nicht um das Politische kümmern. Denn solche Wertfreiheit ist ja nicht zuletzt für das angebliche Verschwinden des Politischen verantwortlich. Zu fordern ist vielmehr, daß die seit den fruihen siebziger Jahren eingeleiteten Bemühungen um eine gesellschaftsbewußte und politisch verantwortliche Naturwissenschaft konsequent weitergeführt werden.

"Politisch verantwortlich" ist indessen nicht gleichzusetzen mit einer impliziten oder gar expliziten Übernahme politischer Verantwortung, die Naturwissenschaftern in letzter Zeit "kraft Wissenschaft" zunehmend übertragen wurde. Darin liegt ein gefährlicher Trugschluß. Eine politisch verantwortungsbewußte Naturwissenschaft charakterisiert sich vielmehr dadurch, daß sie die politisch $z u$ bewertenden Bedingungen und Implikationen ihres Tuns offenlegt und diese den politischen Instanzen zur Entschei- dung unterbreitet. Dies bezieht sich namentlich auf mögliche unterschiedliche Bewertungen von Sicherheitsmargen und auf das Sichtbarmachen der Grenzen ökologischer Beweisführungen. Darüber hinaus sollten die Naturwissenschafter Politik und Verwaltung vermehrt dazu zwingen, ihre Fragestellungen bezüglich der interessierenden Handlungsdimensionen zu präzisieren und zu definieren, welche Qualitätsanforderungen an "intakte" Ökosysteme zu stellen sind.

\section{Zur neuen Rolle der Politik}

Bei der Rehabilitierung des Primats der Politik im "vernaturwissenschaftlichten" Umweltdiskurs ist in allererster Linie die Politik selbst zum Handeln aufgefordert. Denn es geht, um ein Kant-Zitat ${ }^{[29]}$ abzuändern, um nichts weniger als um den Aufbruch der Politik aus ihrer "selbstverschuldeten Unmündigkeit". Politiker aller Schattierungen haben es zugelassen, daß die Rolle der Politik zunehmend marginalisiert wurde und der politische Kern in der Umweltpolitik unter vernaturwissenschaftlichten Gewändern zunehmend verdeckt wurde. Wenn es Aufgabe von Sozial- und Naturwissenschaften ist, diesen politischen Kern wieder sichtbar zu machen, so muß die Politik ihrerseits den ihr durch den Wählerauftrag unzweifelhaft übertragenen Mut zur Verantwortung unter Beweis stellen. Sie kann sich nicht länger hinter den weißen Mänteln der Ärzte, Chemiker oder Physiker verbergen. Wer diesen Mut nicht aufbringt, soll sich nicht wählen lassen!

In diesem Sinne sollten verantwortliche Politiker aus Parlament und Regierung vermehrt die politischen Handlungsdimensionen bezeichnen, die ihren naturwissenschaftlichen Fragestellungen zugrunde liegen; und sie sollten außerdem die Qualitätsvorstellungen klarer formulieren, die die zu schützenden Ökosysteme aufweisen müssen ${ }^{[30 \mid}$. Ferner ist es an den Politikern, die erforderlichen Sicherheitsmargen zu definieren und sich darüber auszusprechen, was sie unter einem "naturwissenschaftlichen Beweis" verstehen. Zu diesem Zweck müssen Politiker vermehrt lernen, "zwischen den Zeilen" wissenschaftlicher Gutachten zu lesen. Skepsis gegenüber "Einervorschlägen" sollte dazu führen, daß Politiker inskünftig noch vermehrt die Ausarbeitung von Varianten verlangen, in denen Ursache-Wirkungs-Gefüge, Bedrohungspotentiale et cetera nach Maßgabe unterschiedlicher Anforderungen an die Beweisführung, unterschiedlicher Sicherheitsmargen oder Qualitäts-

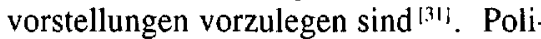


tiker sind nicht dazu gewählt, wissenschaftliche Einervorschläge abzusegnen, sondern die Wählerinnen und Wähler haben sie ausdrücklich auch mit der Qual einer (vernünftigen) Auswahl belastet. Wer bei dieser Auswahl nur jene Ergebnisse zur Kenntnis nehmen will, die seinen eigenen Interessen oder denjenigen seiner Klienten entsprechen, sollte lieber das Geschäft eines ehrlichen Lobbyisten und nicht dasjenige der Politik betreiben.

\section{Ausblick}

Die Wiederentdeckung des Politischen und dessen angemessene Berücksichtigung und Entscheidung durch die Politik ist in den neunziger Jahren ein wichtiger Programmpunkt von Sozial- und Naturwissenschaften, selbstverständlich aber auch der Politik selbst. Dies gilt für jede Stufe staatlicher Entscheidungen, ob diese nun in der Gemeinde, im Kanton, im Bund oder in internationalen Organisationen zu treffen sind. Angesprochen ist nicht nur die planetare Entscheidung über Reduktionsquoten von $\mathrm{CO}_{2}$ Emissionen der einzelnen Länder. Eine Vielzahl angeblich "kleiner" lokaler Entscheidungen hat ebenso wichtige Auswirkungen wie die Entscheidungen der sogenannt "großen" nationalen oder internationalen Politik. Dieses Postulat gilt sowohl für die alten Umweltpolitiken, die diesbezüglich einer neuen Beurteilung zu unterziehen sind, als auch für neue umweltpolitische Strategien etwa der $\mathrm{CO}_{2}$-Emissionen oder einer globalen Umorientierung der traditionellen Umweltpolitik im Sinne einer neuen ökonomischen Ressourcenbewirtschaftungspolitik [32].

\section{Literarurverzeichnis}

[1] Vergleiche dazu G. Winter (Ed.): Grenzwerte. Interdisziplinäre Untersuchungen zu einer Rechtsfigur des Umwelt-, Arbeits- und Lebensmittelschutzes, Umweltrechtliche Studien 1 - Technik, Umwelt, Energie, Recht, Werner, Düsseldorf (1986), insbesondere p. $1 \mathrm{ff}$. (Einführung), p. $73 \mathrm{ff}$. (Entscheidungsprozesse) und p. $227 \mathrm{ff}$. (Strukturen und Alternativen).

[2] Vergleiche dazu VSS/Schweizer Norm Nr. $640080 a$ (genehmigt November 1981 ).

[3] Vergleiche dazu W. Hüsler et al.: Verkehrsflächen der Schweiz, Materialien zur Raumplanung, Eidgenössischen Justiz- und Polizeidepartement, Bundesamt für Raumplanung, Bern (1989)

[4] Volksabstimmung über die Initiative "Pro Tempo 130-100" am 26. November 1989 mit $62 \%$ Nein-Stimmen abgelehnt, Statistisches Jahrbuch der Schweiz 1991, Verlag Neue Zürcher Zeitung, Zürich (1990), p. 349.

[5] Verordnung über die Umweltverträglichkeitsprïfung vom 19. Oktober 1988 (UVPV), SR
814. 011 (in Kraft seit dem 1. Januar 1989).

[6] Vergleiche dazu Bundesamt für Umwelt Wald und Landschaft (BUWAL): Handbuch Umweltverträglichkeitsprüfung ( $U V P$ ), Bern (September 1990)

[7] P. Knoepfel, M. Descloux: "Valeurs limites d'immissions: choix politiques ou determinations scientifiques?", in CERMES, Cahier No 13; Environnement, science et politique GERMES, Paris, (1991), p. 255-280.

[8] Vergleiche dazu R. Häberli, C. Lüscher, B. Paplan Chastonay, C. Wyss: KulturBoden, Boden-Kultur, Vorschläge für eine haushälterische Nutzung des Bodens in der Schweiz, Schlußbericht des Nationalen Forschungsprogrammes (NFP) 22, "Nutzung des Bodens in der Schweiz", Verlag der Fachvereine, Zürich (1991).

[9] Zielvorstellungen für eine erreichbare Reduktion der $\mathrm{CO}_{2}$-Emissionen, Referat $\mathrm{Z}$ III 5, Bonn (13. Juni 1990), p. $15 \mathrm{ff}$

[10] Kabinettsbeschluß vom 13. Juni 1990, Bonn, p. 3, Punkt 1.

[11] Vergleiche dazu Bundesamt für Umwelt, Wald und Landschaft (BUWAL): $\mathrm{CO}, \mathrm{Abga}$ be, Zwischenbericht, Bern (Oktober 1990).

[12] Organisation for Economic Co-operation and Developrment (OECD): Environmental Indicators, A Preliminary Set, Paris (1991), p. $17 \mathrm{ff}$. (Daten für 1988).

[13] Bundesamt für Umwelt, Wald und Landschaft (BUWAL): Umweltbericht $90-$ Zur Lage der Umwelt, Bern (1991), p. $82 \mathrm{ff}$

[14] Vergleiche dazu P. Knoepfel, W. Zimmermann: Ökologisierung von Landwirtschaft - Fünf Geschichten und eine Analyse, Sauerländer, Aarau. ( 1987), p. 68.

[15] Ein Einbezug der gesellschaftlichen Ursachen wird im Rahmen der schweizerischen Umweltbeobachtung angestrebt. Vergleiche dazu: Arbeitsgruppe Umweltbeobachtung der Schweizerischen Naturforschenden Gesellschaft (SNG): Umweltbeobachtung Schweiz, Programm für eine integrierte ökologische Raumbeobachtung, Bern (1987), p. 14 ff.; Schweizerische Kommission für Umweltbeobachtung (SKUB): Zwischenbericht 1991, Bem (1991)

[16] Vergleiche dazu immer noch L. Veuve: La planification iterative, Dossier de travail du cours de perfectionnement en politique de l'environnement a l'IDHEAP (module N 9), polycopie, Lausanne (1987).

[17] Vortrag vom 3. Juni 1989 am Symposium der Schweizerischen Vereinigung für Politische Wissenschaft zum Thema "Politikwissenschaften und Naturwissenschaften" in Solothurn (Manuskript 1989)

[18] »Le risque est-t-il une "construction sociale" ? $\star$ Dazu: J.-L. Fabiani, J. Theys (Ed.): La société vulnérable, évaluer et mâitriser les risques, Presse de l'école normale supérieure, Paris (1987), p. $89 \mathrm{ff}$.

[19] Zum Begriff Restrisiko siehe: R. Coppock: "Risk Analysis in the United States and the United Kingdom", in P. Knoepfel (Ed., im Auftrag der SAGUF): Risiko und Risikomanagement, Reihe Ökologie \& Gesellschaft, Band 2, Helbing \& Lichtenhahn, Basel (1988), p. $85 \mathrm{ff}$.

[20] G. Strey: Umweltethik und Evolution Vandenhoek \& Ruprecht, Göttingen, (1989), p. $60-70$.

[21] A. Schnaiberg: "Discussion: The role of experts and mediators in the channelling of distributional conflicts", in A. Schnaiberg, N. Watts, K. Zimmermann (Ed.) Distributional Conflicts in Environmental-
Resource Policy, WZB Publications, Gower, Hants (1986), p. $348 \mathrm{ff}$

[22] Übersicht bei K. Tobias: Konzeptionelle Grundlagen zur angewandten Ökosystemforschung, E. Schmidt, Berlin (1991), p. 40 ff.

[23] M. Gough: "Incertitude scientifique et instru mentalisation politique. La polemique sur l'agent orange et la dioxine aus Etats-Unis", in GERMES 1991, Ref. [7], p. 239-254.

[24] D. Freiburghaus, W. Zimmermann: "Wie wird Forschung politisch relevant? Erfahrungen in und mit den Nationalen Forschungsprogrammen", Publikationen des Schweizerischen Nationalfonds, Nr. 28, Haupt, Bern (1985), p. 33-120 (Beispiele).

[25] Vergleiche dazu Ref, [14], p. $158 \mathrm{ff}$

[26] Zwar gibt es viele immer wieder zitierte Äußerungen großer Naturwissenschaftler zur politischen Verantwortung (etwa von A. Einstein, C. F. von Weizsäcker, J. R. Oppenheimer im Zusammenhang mit der Entdeckung der Atomspaltung). Das Thema wird indessen in der politischen Ethik noch kaum behandelt. Hinwcise auf die politischen Konsequenzen der ökologischen Krise finden sich bei a) V. Hösle: Philosophie der ökologischen Krise, Moskauer Vorträge, C. H. Beck, München (1991), p. 121-146;

b) E. Shargaff: "Allmacht und Ohnmacht der Naturwissenschaften - Bemerkungen zur Wissensindustrie unserer Zeit", in R. Löw et al. (Ed.): Fortschritt ohne Maß, eine Ortsbestimmung der wissenschaflich-technischen Zivilisation, München (1991), p. 13-25.

[27] Ausfuihrungsplan, Schweizerischer Nationalfonds zur Förderung der wissenschaftlichen Forschung: NFP $3 I-$ Bern (1991), p. $3 \mathrm{ff}$.

[28] Das wird indessen an Studenten der Politikwissenschaften selten vermittelt.

[29] I. Kant: "Beantwortung der Frage: Was ist Aufklärung?", in W. Weischedel (Ed.).: Schriften zur Anthropologie, Geschichtsphilosophie, Politik und Pädagogik, Erster Teil, Band 9. Werke in zehn Bänden, Wissenschaftliche Buchgesellschaft, Darmstadt (1975), p. 53.

[30] Ein gutes Beispiel geben Artikel 13 und 14 des Schweizerischen Umweltschutzgesetzes vom 7. Oktober 1983 (SR 814). Danach ist das Schutzobjekt der Immissionsgrenzwerte nicht auf die menschliche Gesundheit beschränkt, sondern es umfaßt auch Tiere, Pflanzen, Lebensgemeinschaften, Lebensräume oder die Fruchtbarkeit des Bodens; außerdem sollen auch die menschlichen Risikogruppen einbezogen werden. Ob sich allerdings die Politiker der Konsequenzen dieser Bestimmung für die relativ strenge Luftreinhaltepolitik bewußt waren, läßt sich angesichts der Auseinandersetzungen über die Bedeutung der Grenzwerte heute füglich bezweifeln.

[31] Wie das in vorbildlicher Weise etwa im Luftreinhalte-Konzept des schweizerischen Bundesrates vom 10. September 1986 erfolgte.

[32] Siehe dazu The World Commission of Environment and Development: Our Common Future, Oxford University Press, Oxford (1987), p. $43 \mathrm{ff}$ und $261 \mathrm{ff}$; Union mondiale pour la nature (UICN), Programme des Nations Unies pour l'environnement (PNUE), Fonds mondial pour la nature (WWF): Sauver la Planéte, Strategie pour l'Avenir de la Vie, Glands (1991), p. 99ff. 